\title{
Observations of plants with tubular stems with possible biomimetic implications
}

\author{
Masaya Goto ${ }^{1}$, Aohan Wang ${ }^{2}$, Hiromasa Goto ${ }^{2}$ \\ 'Joso Gakuin, 1010 Nakamuranishine, Tsuchiurashi, Ibaraki, 300-0849 Japan \\ ${ }^{2}$ Graduate School of Pure and Applied Sciences, University of Tsukuba, Tsukuba, Ibaraki 305-8573, \\ Japan \\ E-mail: m_research@outlook.jp (M. Goto), \\ s-awang@ims.tsukuba.ac.jp (A. Wang), gotoh@ims.tsukuba.ac.jp (H. Goto)
}

Keywords: Butterbur; Water lily; Layers; Stem; Optical fiber

\begin{abstract}
Two plants having tubular stems were examined. Water lily belongs to nymphaeaceae, while butterbur belongs to asteraceae. These plants are not relatives; however both have large leaves with lacinia. Furthermore, these plants have tubular long stems. Observations of leaf surface are described. Water lily leaf has an inside layer consisting of a network polygonal structure. The leaf has three layers at the microscopic level including small transparent skin tissue, and a middle layer showing a polygonal green structure where photosynthesis can be performed. The bottom layer shows fine tubular tissues. Stomas are observed on the surface of the leaf, with transparent tissue on the top layer and the stem of the butterbur with honeycomb (polygonal) structures in the leaves and stems. Plants are of interest for technology because their remarkable functional structure may provide clues for applications in materials science. In this study, the fine structures of the two plants were observed to learn their functional structure.
\end{abstract}

\section{INTRODUCTION}

Water lily (nymphaeaceae) shows similarities with lotus although a different species. The leaf is highly distinctive with photosynthesis carried out by the leaf floating on the water. Oxygen and carbon dioxide can be sent to roots beneath the water surface through an air duct in the stem. In this study, we observed the characteristic surface of the water lily and discuss the function of the leaf.

Butterbur has large leaf, similar in appearance to that of the water lily. The tubular stem of the butterbur provides mechanical strength and flexibility. Furthermore, the stem can send gases toward roots. This function may allow it to perform internal photosynthesis.

Plants are of interest for technology because their remarkable functional structure may provide clues for applications in materials science. In this study, the fine structures of the two plants were observed to learn their functional structure. 


\section{RESULTS AND DISCUSSION}

\subsection{Water lily}

\subsubsection{Optical microscopy}

Water lily has many similarities to lotus. We observed water lily tissue with an optical microscope, and discuss possibility of light collection and transmission function.

Figure 1 shows sample preparation (A) and optical microscopy image (B, C). This observation revealed that the water lily leaf has an inside layer consisting of a polygonal network structure (collenchyma). Figure 2 displays optical microscopy images of the water lily leaf (Figure 2). The leaf consists of three microscopic layers. Small transparent skin tissue was observed, as shown in Figure 2B.

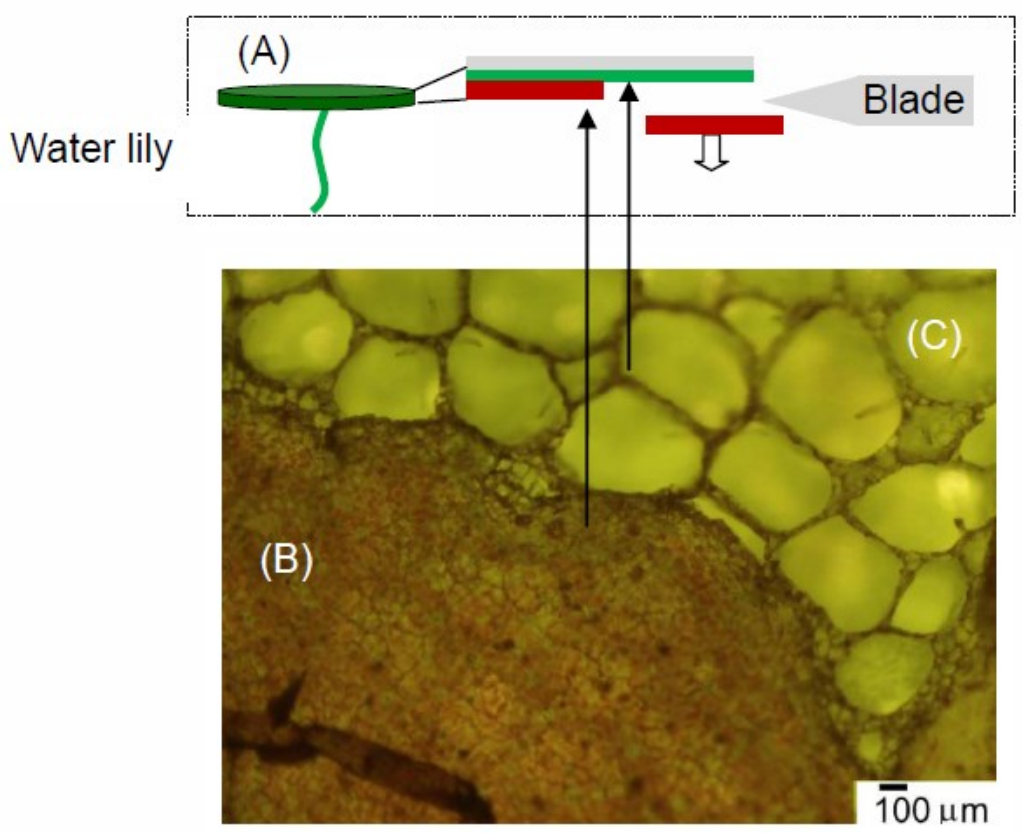

Figure 1. Optical microscopy image of water lily leaf observed from the backside. (A) sample preparation; (B) Surface of backside of the leaf; (C) interior of the leaf.

The interior layer (middle layer) shows polygonal green structure. Photosynthesis can be performed in this layer (Figure 2C). The bottom layer shows fine thread like tissues (Figure 2D). As seen in Figure 2E and Figure 3, stomas are observed on the surface [1]. In general, stomas are located on the backside of the leaves in plants. However, water lily has stomas on the surface, as shown in Figure 2E, obtained with the replica method (Suzuki's Universal Micro-Printing (SUMP) method). This may be due to the fact that the backside of the leaf faces the water. Water lily breathes on the surface of the leaf in contact with the air. 


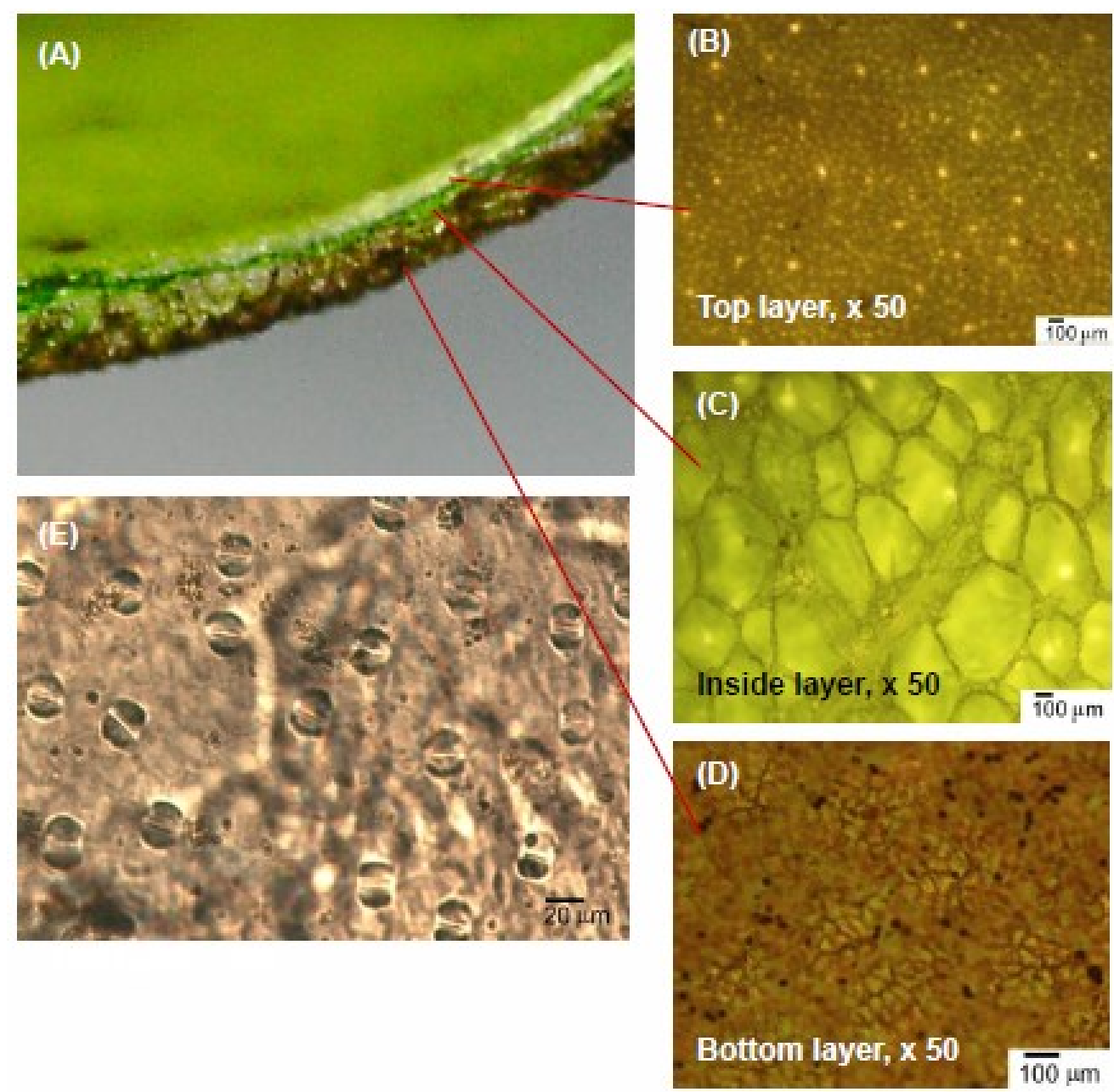

Figure 2. (A): Optical microscopy image of surface, inside, and bottom layer of the water lily leaf. (B): Top layer. (C): inside layer (middle layer). (D): Bottom layer. (E): Magnification image of the top layer appears stomas. Surface image of the water lily leaf obtained by the SUMP replica method with differential interference contrast optical microscopy.

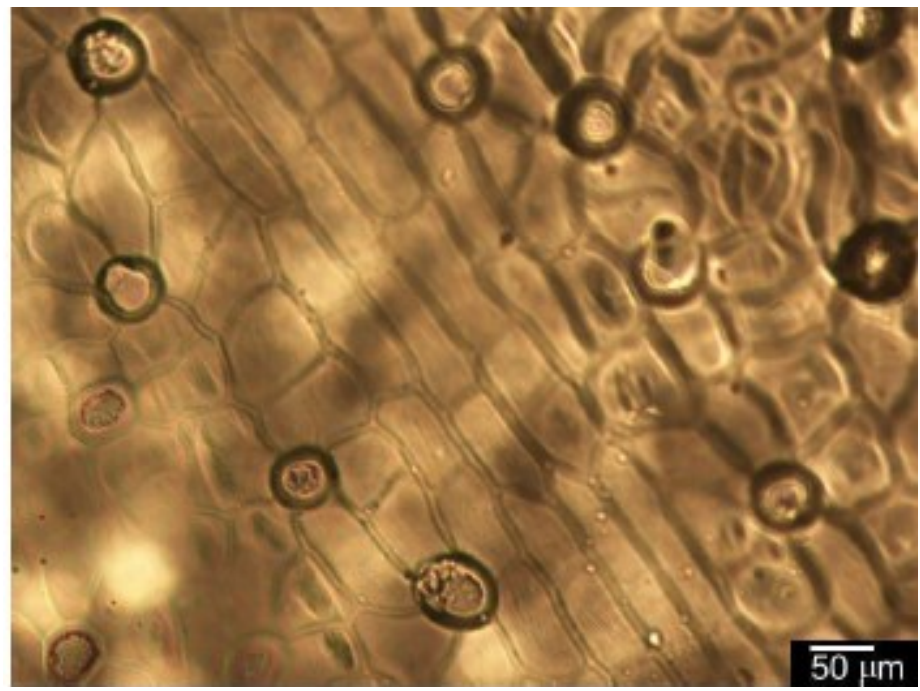

Figure 3. Bottom layer image of the water lily leaf. The image was obtained by the SUMP replica method with a differential interference contrast optical microscopy. 


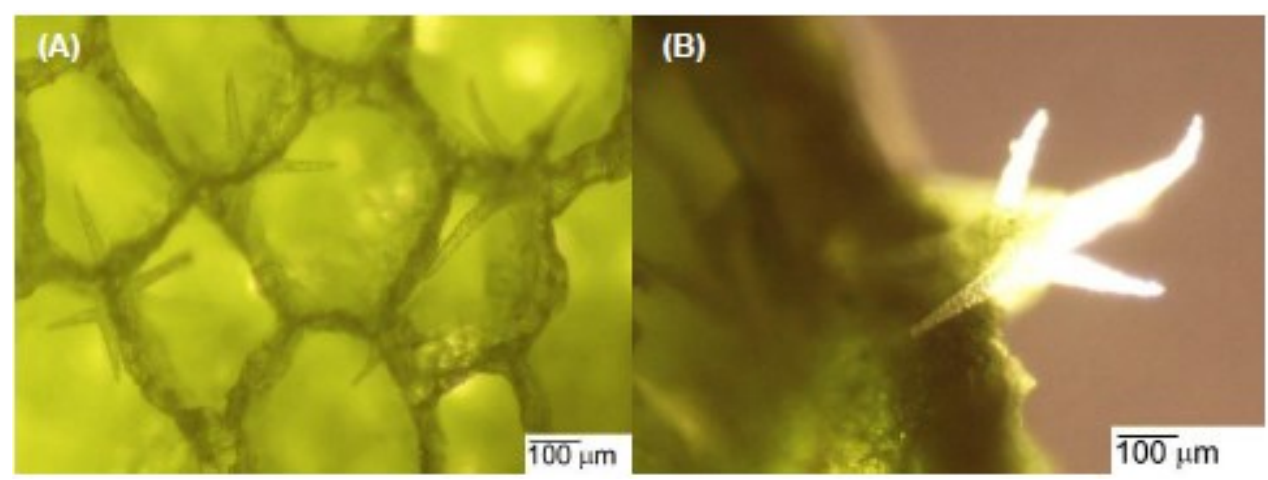

Figure 4. Star-shaped cells (astrosclereid) based in a nodal cell of aerenchymatic tissue.

Note that star-shaped cells (astrosclereid) are observable at the nodal cell of aerenchymatic tissue, as shown in Figure 4A [2,3]. Figure 4B displays the star-shaped cells. The foliar sclereids Olea europaea may function as optical fibers to improve the light micro-environment [4]. The small dots on the star-shaped cell surface are crystals having high calcium content evaluated with SEM photograph and X-ray map [5]. The structure reinforces the leaf through the polygonal form. Both the polygonal and star-shaped structures contribute to the light collection and mechanical strength. Furthermore, the polygonal structure decreases the density allowing the leaves to float. Functions of the polygonal structure in the leaf are summarized as 1) light collection, 2) mechanical strength, and 3) floatation.

\subsubsection{Optical absorption}

Chlorophylls absorb both red and blue light range to perform photosynthesis. Optical absorption spectrum of the green solution in ethanol extracted from water lily stem shows absorption at purple-blue and red wavelength. Green optical absorption is weak (Figure 5). Water lily stem appears green as a complementary color of blue and red. Note that water lily stem above or below the water shows green. This implies that photosynthesis can perform at the stem.

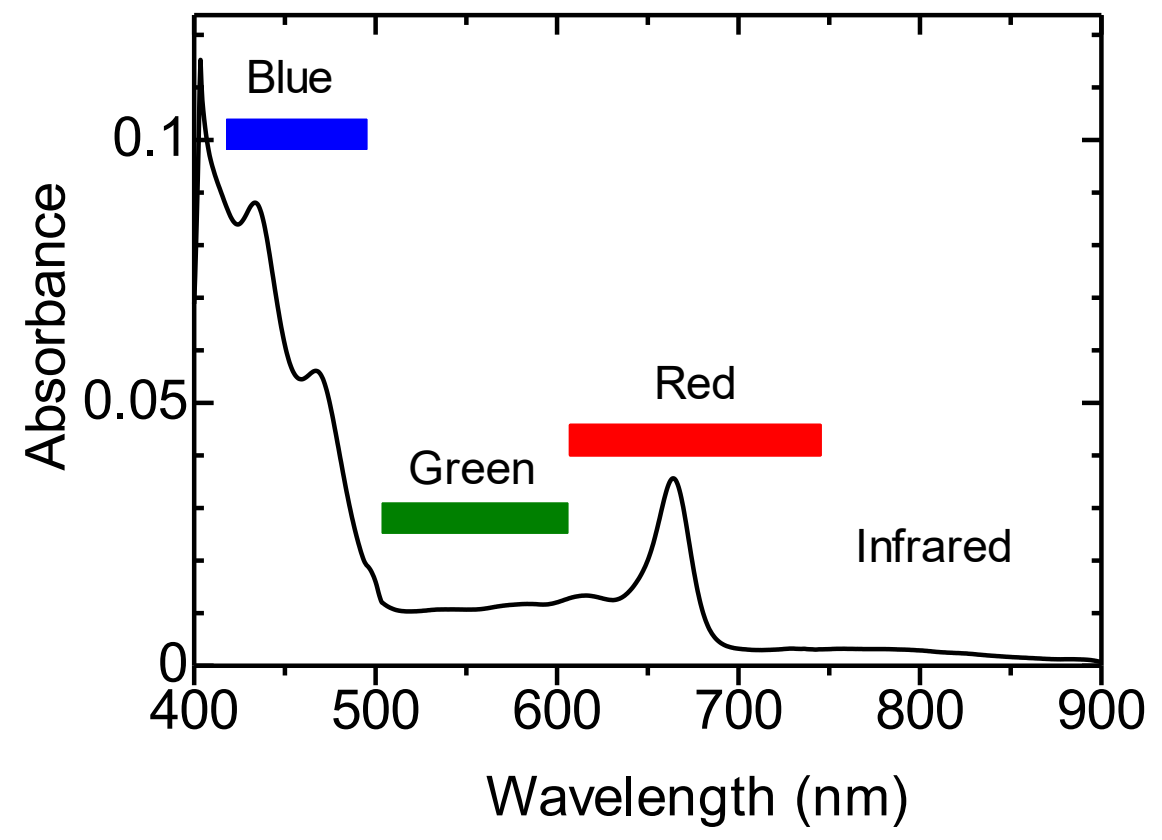

Figure 5. Optical absorption of a green solution in ethanol extracted from water lily stem. 


\subsubsection{Possibility of remote photosynthesis}

The surface cleaning system of the papillae can perform effectively for light collection. The polygonal structure contributes mechanical strength to maintain the leaf open in the sun. Air duct and textile tissues may transmit light. Internal photosynthesis from distance, which is referred to as "remote photosynthesis" may occur.

\subsubsection{Hierarchical structure}

Figure 6A shows water lily leaves on the water. Figure 5B displays the backside of the leaf. Radiation-like vein is observable (magnified image shown in Figure 6C, with schematic in 6D), indicating that the leaf consists of polygonal structures in the leaf vein and at the cell level. These structures can provide strength and floatation for the leaf on the water.

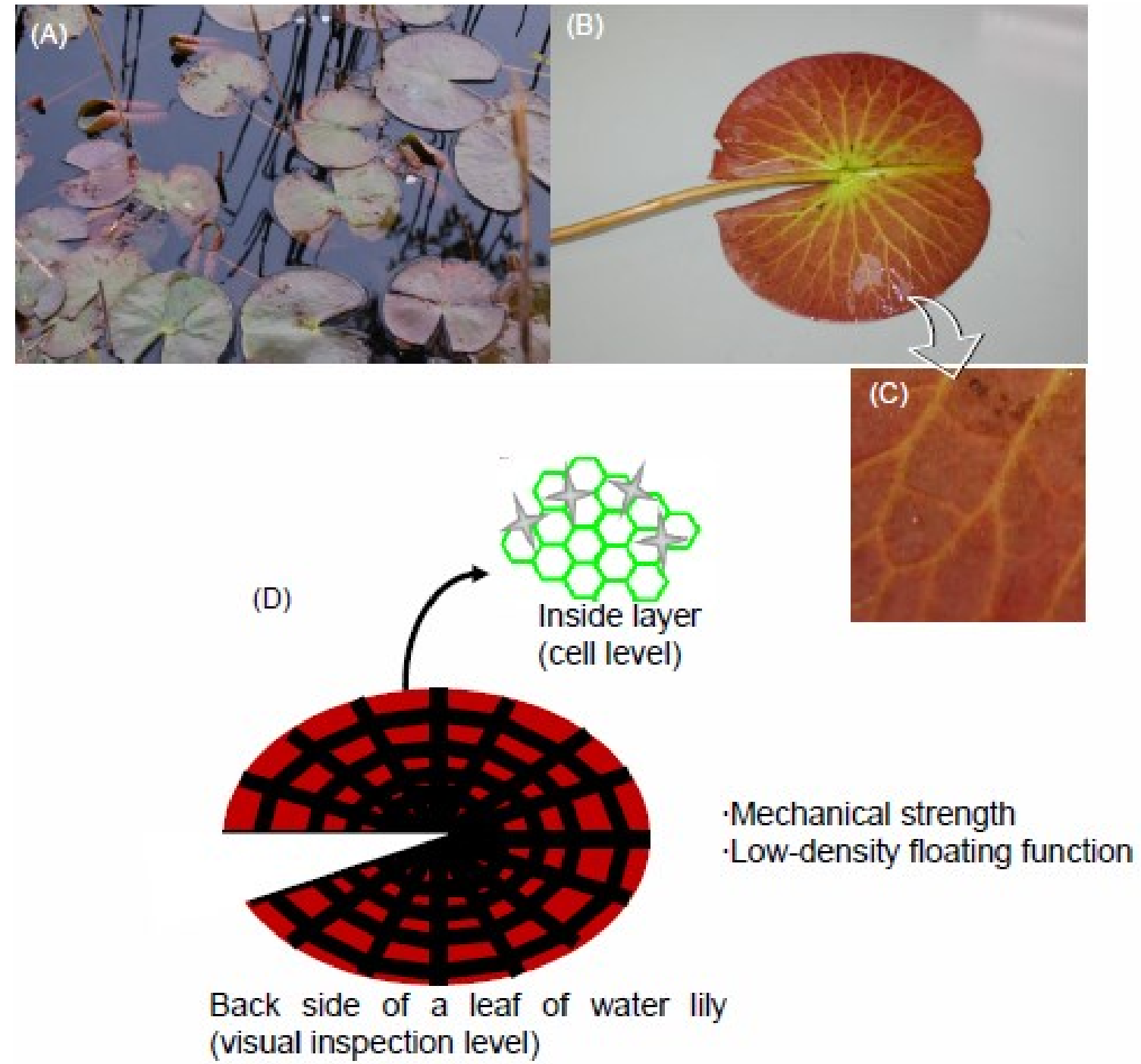

Figure 6. Hierarchical structure. (A): Water lily. (B): Back side of leaf. (C) Magnified image. (D): Illustration of backside of the leaf and inside layer. 


\subsection{Butterbur}

\subsubsection{Observations of butterbur}

The surface structure of petasites japonicas (butterbur) stem was observed under polarizing optical microscope with different magnifications (Figures 7,8B). Figure 8A shows an illustration image of the cross section, where a hollow tube at the center was observed in the middle part. Vascular bundles are located on both inner and outer peripheries. A notch was observed on the leaf. Inside of stem is filled with cells, for example, Figure 8C shows the structure near the hollow tube. Then honeycomb structure (foam like structure) cells in the stem can help resist kinking when mechanically bent [8]. The structure endows strength and flexibility. On the outer circumference we observed a three-layer structure of epidermis and cortex (collenchyma and parenchyma) (Figure 8D). By peeling away the epidermis very carefully a very thin layer was obtained that appeared as a single layer with honeycomb structure (Figure $8 \mathrm{E}$ ). Figure $8 \mathrm{~F}$ is a magnified image of a vascular bundle cross section. All the pictures were observed under fluorescent light $(450-490 \mathrm{~nm})$ to reveal the structure clearly.

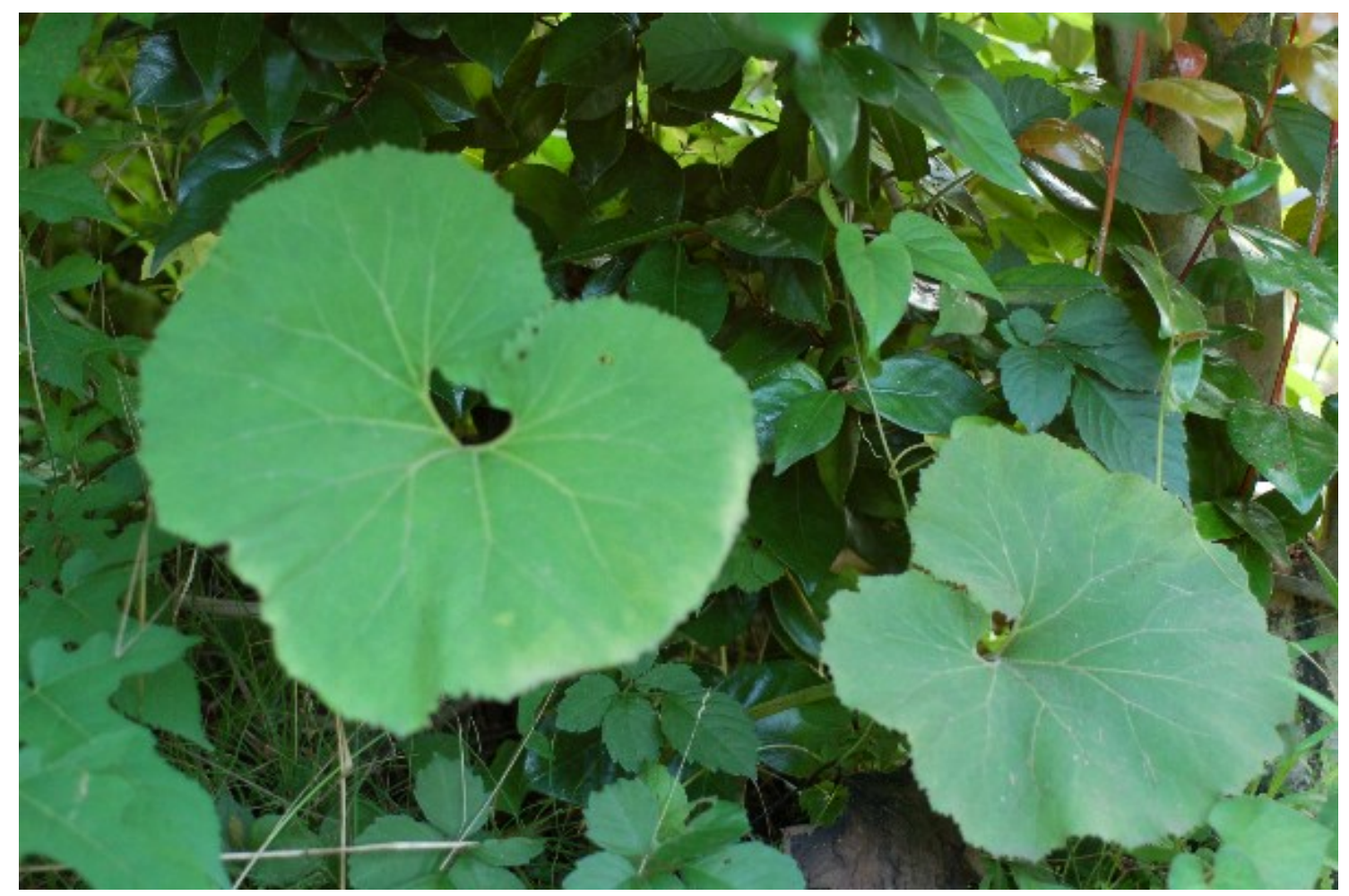

Figure 7. Butterbur. 

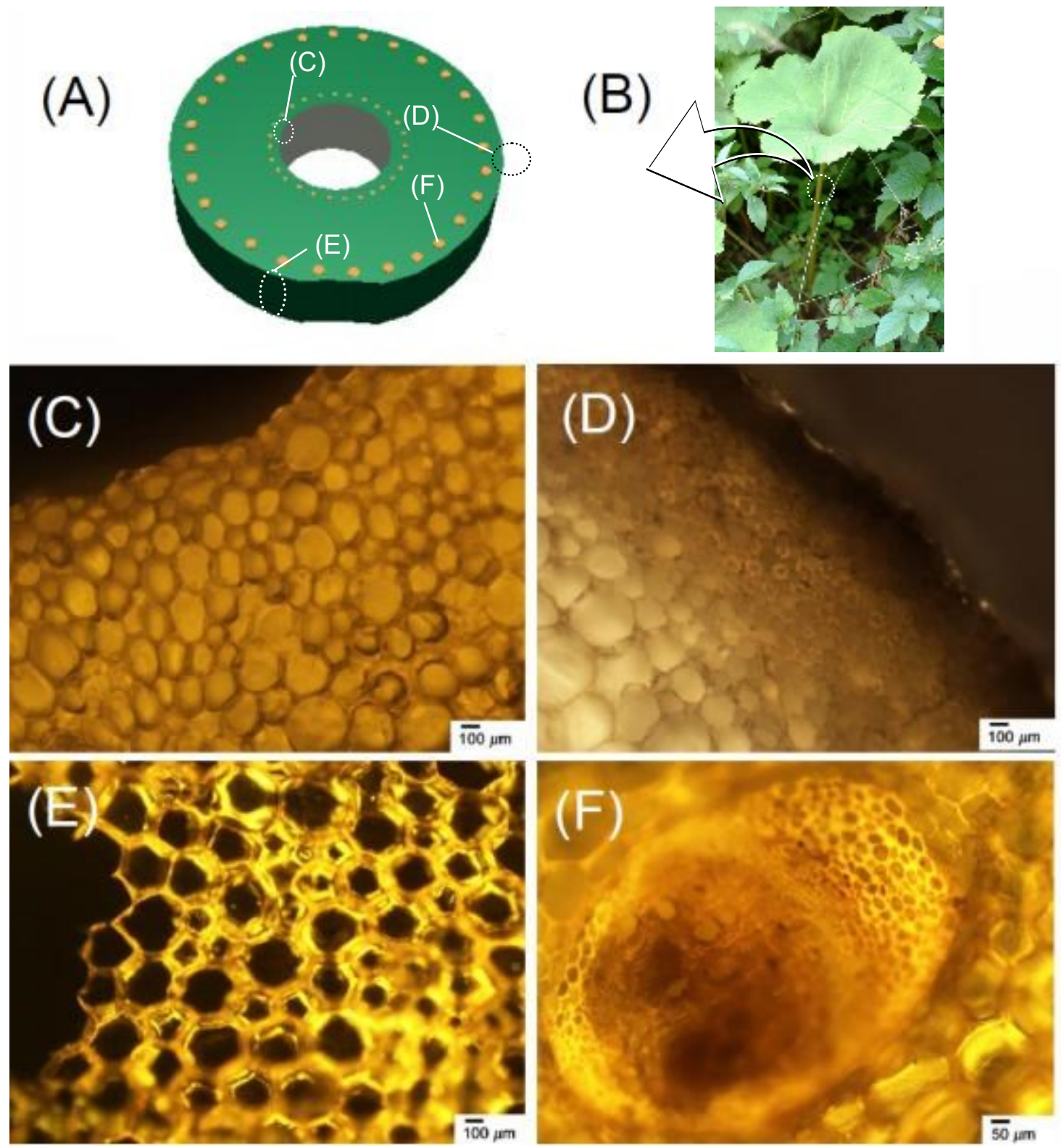

Figure 8. (A): An illustration of stem cross section $(\times 50)$. (B): Petasites japonicas (butterbur). (C): Cells near the tube. $(\times 100)(\mathrm{D})$ : Epidermis and cortex (collenchyma and parenchyma). (E): Honeycomb structure of cells peeled to a thin layer. $(\times 50)$. $(F)$ : Magnified mage of vascular bundle $(\times 100)$. All the pictures were observed under fluorescent light $(450-490 \mathrm{~nm})$ in order to reveal the structure clearly. (C)-(D): Polarizing optical microscopy images. 

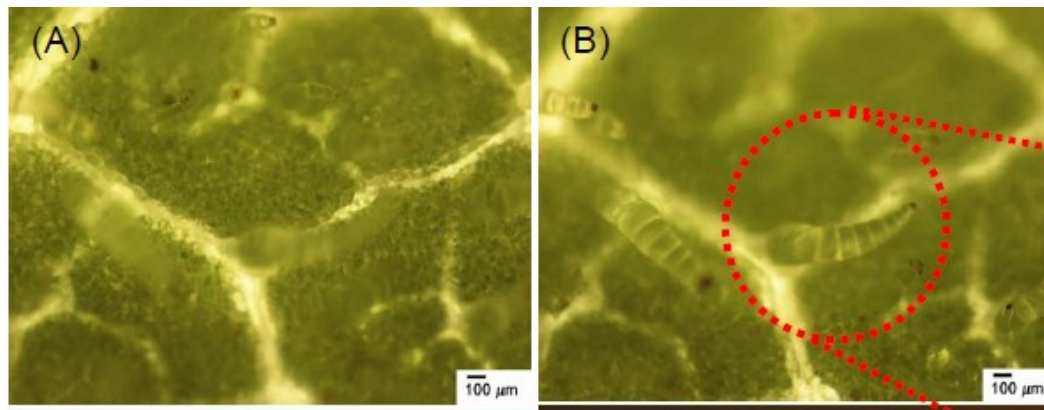

(D)
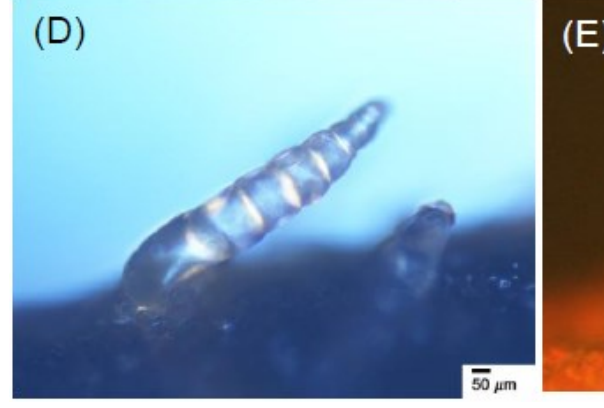

(E)

Figure 9. Polarizing optical microscope images of petasites japonicas leaf surface. (A): Top layer $(\times 50)$. (B): Same observation field of $(\mathrm{A})$ with different focus distance $(\times 50)$. $(\mathrm{C})$ : Magnification image of top layer $(\times 100)$. (D), (E): Side-view images of petasites japonicas leaf $(\times 100)$. Worm-like structural projections were found on leaf surface. (E): Fluorescence microscopy image.

\subsubsection{Possibility of optical waveguide}

Surface structure of the leaf was also observed under polarizing optical microscopy. Figures 9A and 9B show the leaf surface with different focus. A transparent membrane was observed on the top layer. Figure 9C displays the worm-like structural projections located on the leaf surface, which could be found throughout the top layer that look like a white fiber. To observe the image clearly, lights were irradiated from top and bottom direction to the sample (Figure 9D), while Figure 9E was obtained with fluorescence optical microscopy (excitation wavelength $=450-490 \mathrm{~nm}$ ). The transparent membrane and worm-like structure may be related to the light collection system, as suggested in Figure 10 (possible mechanism). The surface transparent fibers as a light collector transmit light to the inside of leaf [6].

Euplectella (Animalia) is a marine organism having fiber optical properties [7]. Transparent life form can function as optical waveguides. Plants may make use of transparent tissue on the leaf for effective photosynthesis. 


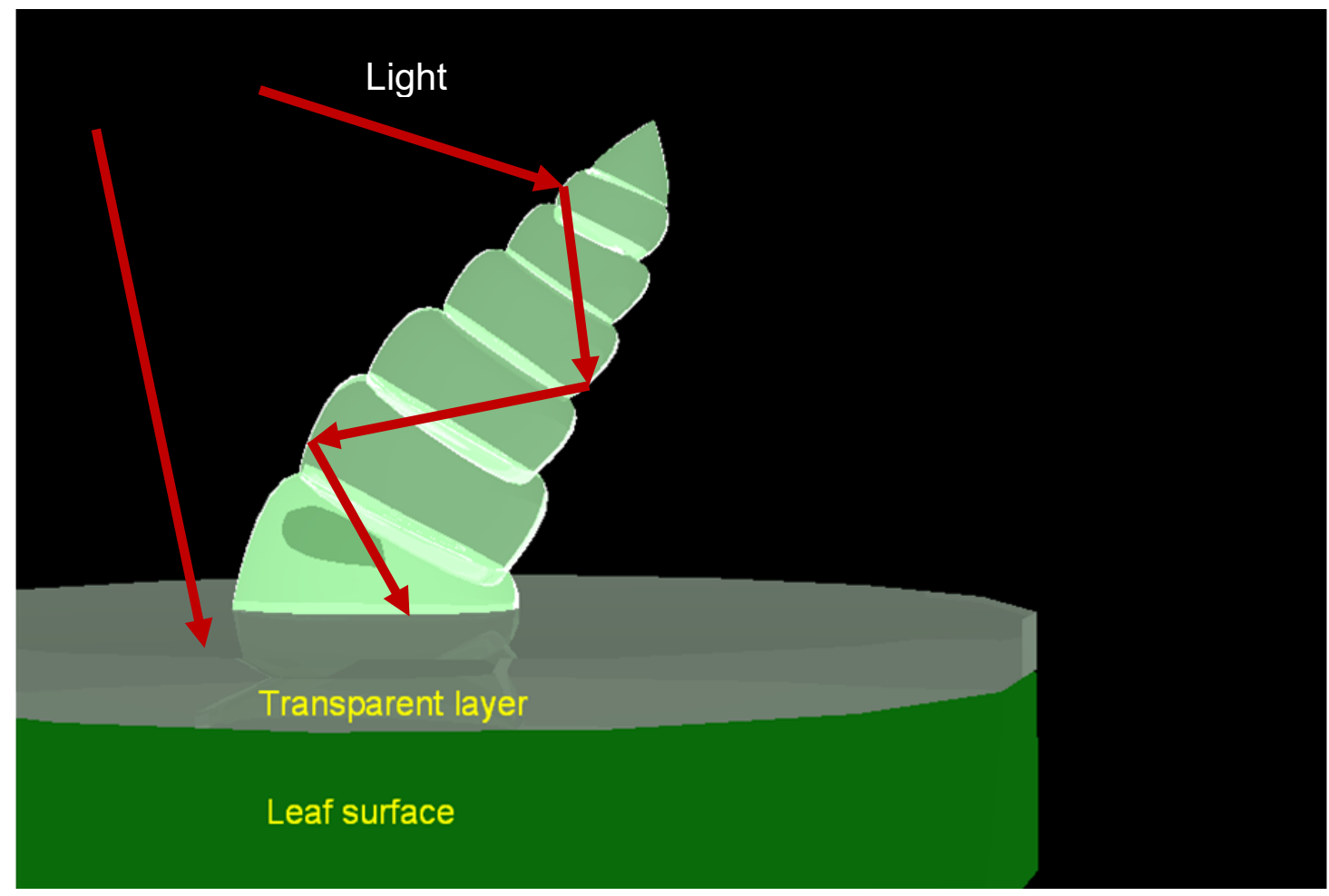

Figure 10. Possible mechanism of light collection of the leaf.

\section{CONCLUSIONS}

Water lily leaf has three microscopic layers including thin transparent skin tissue. The interior layer (middle layer) shows polygonal green structure where photosynthesis is performed. Stomas are observed on the surface of the leaf. Star-shaped cells (astrosclereid) are observable at the nodal cell of aerenchymatic tissue. The hierarchical structure provides mechanical strength and low-density floatation. Butterbur also has tubular stems whose tissues consist of a polygonal structure (bubble-like). Although the two plants are different species, similar function and structure has evolved. Transparent surface worm-like tissue may perform light collection as biological optical fibers. We can learn water-floating function, flexibility, strength of stem derived from polygonal structure (foam like structure), and optical functions of the plants for applications in technology.

\section{TECHNIQUE}

Optical observations were carried out using an ECLIPS LV 100 high-resolution polarizing microscope (Nikon) with a LU Plan Fluor lens and a CFIUW lens (Nikon). UV-vis absorption spectra were recorded on a V-630 UV-vis optical absorption spectrometer (JASCO).

\section{SAMPLES}

The water lily and butterbur samples were obtained in Tsukuba in 2015.

\section{CONFLICTS OF INTEREST}

The authors declare no conflict of interest. 


\section{References}

[1] Qiu, F., Spring, A. M., Maeda, D., Ozawa, M., Odoi, K., Aoki, I., Otomo, A., Yokoyama, S. A., Straightforward Electro-optic Polymer Covered Titanium Dioxide Strip Line Modulator with a Low Driving Voltage, Appl. Phys. Lett., 105 (2014)073305.

[2] Schweingruber, F. H., Börner, A., Schulze, E.-D., Atlas of stem anatomy in herbs, shrubs and trees, Berlin, Springer, 1 (2011) 286-289.

[3] Inoh, S., Plant Histology, Uchida rokakuhosinsha (Japanese), Tokyo, P109 (1964).

[4] Karabourniotis, G., Papastergiou, N., Kabanopoulou, E., Fasseas, C., Can. J. Bot., 72 (1994) $330-336$.

[5] Kuo-Huang, L.-L., Chen, S.-H., Chen, S.-J., Bot. Bull. Acad. Sin. 41 (2000) 283-291.

[6] Goto, M., Wang, A., Goto H., submitted.

[7] Sundar, V. C., Yablon, A. D., Grazul, J. L., Ilan, M., Aizenberg, J., Fibre-optical features of a glass sponge, Nature, 424 (2003) 899.

[8] Gibson, L. J., Inside Plants: An Engineer's View of the Arnold Arboretum, Arnoldia, 70 (2012) 11-19. 